\title{
On Minimum Stars and Maximum Matchings*
}

\author{
S. P. Fekete ${ }^{1}$ and H. Meijer ${ }^{2}$ \\ ${ }^{1}$ Department of Mathematics, TU Berlin, \\ 10623 Berlin, Germany \\ fekete@math.tu-berlin.de \\ 2 Department of Computer Science, Queen's University, \\ Kingston, Ontario K7L 3N6, Canada \\ henk@cs.queensu.ca
}

\begin{abstract}
We discuss worst-case bounds on the ratio of maximum matching and minimum median values for finite point sets. In particular, we consider "minimum stars," which are defined by a center chosen from the given point set, such that the total geometric distance $L_{S}$ to all the points in the set is minimized. If the center point is not required to be an element of the set (i.e., the center may be a Steiner point), we get a "minimum Steiner star" of total length $L_{S S}$. As a consequence of triangle inequality, the total length $L_{M}$ of a maximum matching is a lower bound for the length $L_{S S}$ of a minimum Steiner star, which makes the worst-case value $\rho(S S, M)$ of the value $L_{S S} / L_{M}$ interesting in the context of optimal communication networks. The ratio also appears as the duality gap in an integer programming formulation of a location problem by Tamir and Mitchell.

In this paper we show that for a finite set that consists of an even number of points in the plane and Euclidean distances, the worst-case ratio $\rho(S, M)$ cannot exceed $2 / \sqrt{3}$. This proves a conjecture of Suri, who gave an example where this bound is achieved. For the case of Euclidean distances in two and three dimensions, we also prove upper and lower bounds for the worst-case value $\rho(S, S S)$ of the ratio $L_{S} / L_{S S}$, and for the worst-case value $\rho(S, M)$ of the ratio $L_{S} / L_{M}$. We give tight upper bounds for the case where distances are measured according to the Manhattan metric: we show that in three-dimensional space, $\rho(S S, M)$ is bounded by $\frac{3}{2}$, while in two-dimensional space $L_{S S}=L_{M}$, extending some independent observations by Tamir and Mitchell. Finally, we show that $\rho(S, S S)$ is $\frac{3}{2}$ in the two-dimensional case, and $\frac{5}{3}$ in the three-dimensional case.
\end{abstract}

\footnotetext{
* Parts of this work were done while the first author was visiting Queen's University, partially supported by the Deutsche Forschungsgemeinschaft, FE 407/3-1. Parts of this work were done while the second author was visiting Universität zu Köln, partially supported by NSERC. A preliminary extended abstract of this article, titled "On minimum stars, minimum Steiner stars, and maximum matchings," appears in the Proceedings of the 15th ACM Symposium on Computational Geometry [9].
} 


\section{Introduction}

The problem of finding a maximum weight matching for a given set of vertices in a weighted graph is to find a set of disjoint edges, such that the total weight of all the edges is maximized. Determining an optimal matching is a classical algorithmic problem, and Edmonds's famous polynomial-time algorithm [7] is one of the milestones of combinatorial optimization.

On the other hand, it has been known for quite a while [11] that the task of finding a minimum weight Steiner tree is an NP-hard problem: find a network of smallest total length $L_{S T}$ that connects all given points, while allowing additional "Steiner" points for connecting edges. This algorithmic intractability differs drastically from the case where no Steiner points are allowed, so that the connected network has to be a minimum weight spanning tree (MST) of weight $L_{T}$, which can be solved very efficiently. Many aspects of optimal Steiner trees have been considered; see the book [13] for an overview. One of the most famous problems related to geometric Steiner trees deals with the worst-case value $\rho(T, S T)$ of the ratio $L_{T} / L_{S T}$. As Du and Hwang [6] managed to prove for the case of planar point sets with Euclidean distances, $\rho(T, S T)=$ $2 / \sqrt{3}$.

A special type of Steiner tree problems arises in the context of location theory: The so-called Weber problem asks for the location of a single center point, such that the sum of distances from the given points to the center is minimized. (See [12]. [5] gives an overview and an extensive list of references.) It was shown by Bajaj [1] that even for the simple case of five points in the Euclidean plane, a solution can in general not be expressed by radicals. (In particular, it is impossible to construct an optimal solution by means of ruler and compass.) In the context of communication networks, the resulting tree has been called a star [10]. As in the case of general tree networks, we can distinguish the Steiner case (where the center point can be chosen anywhere) from the more restricted case, where the center point is required to be chosen from the given set. In the following we speak of "minimum Steiner stars" (with a total edge length denoted by $L_{S S}$ ) and "minimum stars" (with a total edge length denoted by $L_{S}$ ).

When dealing with algorithmically hard problems like the task of designing optimal communication networks, it is of great importance to provide good upper and lower bounds for an optimal solution. It has been pointed out by Fingerhut et al. [10] that $L_{M}$ is a lower bound for $L_{S S}$, which is an upper bound for $L_{S T}$. This makes it interesting to consider the worst-case value $\rho(S S, M)$ of the ratio $L_{S S} / L_{M}$. It was conjectured by Suri [16] that for the case of points in the plane with Euclidean distances, $\rho(S S, M)$ is $2 / \sqrt{3}$ - the Steiner tree ratio. Proving this conjecture is one of the main results of this paper. In addition, we consider the behavior of the worst-case bounds $\rho(S, S S)$ for $L_{S} / L_{S S}$, and $\rho(S, M)$ for $L_{S} / L_{M}$. The ratio $L_{S} / L_{S S}$ appears in location problems, where the sum of distances from a depot to a set of positions is to be minimized. (See the book by Drezner [5] for an overview in location theory.) If the depot may be chosen arbitrarily, we get total cost $L_{S S}$. If the depot has to be chosen from the given set of positions, we get a cost of $L_{S}$. Thus, the worst-case ratio can be interpreted as a bound on the possible payoff from opening a new depot.

For the case of Euclidean distances in two and three dimensions, we prove upper and lower bounds for all these worst-case values. 
The above problems are also of interest when distances are not measured according to the Euclidean metric. Of particular relevance is the case of rectilinear (or "Manhattan") distances, which arises in the context of VLSI layout. Tamir and Mitchell [17] have considered $\rho(S S, M)$ for the case of rectilinear distances, motivated by questions from cost allocation for cooperative matching games. They prove that in the case of points in the plane, $L_{S S} / L_{M}=1$, which implies that the core of a related matching game is nonempty. By establishing a tight upper bound on $\rho(S S, M)$, we can establish the largest possible value of the duality gap for their integer programming formulation for the case of Euclidean distances. In Section 5 we prove that, for the case of Manhattan distances, $\rho(S S, M)=\frac{3}{2}$ and $\rho(S, S S)=\frac{3}{2}$. Section 6 deals with Manhattan distances in three-dimensional space. We show that $\rho(S S, M)=\frac{3}{2}, \rho(S, M)=\frac{5}{3}$, while $\rho(S, M)$ lies between $\frac{5}{3}$ and 2. (See Table 1 in Section 7 for an overview.)

Finally, we would like to note some further algorithmic implications: The result by Tamir and Mitchell [17] yields an $O(n)$ time algorithm for finding a maximum weight matching for a planar point set with Manhattan distances. With some extra work, some of the underlying properties of minimum Steiner stars have been used by Fekete [8] to construct an $O(n)$ time algorithm for finding a traveling salesman tour of maximum total length. (See the paper by Barvinok et al. [2] for more results on this problem.)

The rest of this paper is organized as follows. In Section 2 we introduce some basic notation and some general results. Section 3 deals with Euclidean distances in twodimensional space, while Section 4 contains results for the case of Euclidean distances in three-dimensional space. In Sections 5 and 6 we consider Manhattan distances in two- and three-dimensional space. The concluding Section 7 contains a discussion of remaining open problems.

\section{Preliminaries}

Let $G=(V, E)$ be a graph with nonnegative edge weights $w(e)$. Throughout this paper the vertex set $V$ of $G$ is represented by a point set $P=\left\{p_{0}, p_{1}, \ldots, p_{n-1}\right\}$ from Euclidean space, and edge weights correspond to geometric distances, according to some metric. A star of $P$ is a set of $n-1$ edges (represented by line segments) connecting an element of $P$ with all other elements of $P$. A Steiner star of $P$ with center point $c$ is a set of $n$ edges (represented by line segments), connecting each point of $P$ to $c$. A (perfect) matching of $P$ is a set of $n / 2$ edges that pair each point of $P$ with another unique point of $P$. In the remainder of this paper, any star, Steiner star, or matching is assumed to be a star, Steiner star, or matching of $P$, denoted by the symbols $E_{S}, E_{S S}$, and $E_{M}$. Their lengths are denoted by $\left\|E_{S}\right\|,\left\|E_{S S}\right\|$, and $\left\|E_{M}\right\|$, for a specified metric $\|\cdots\|$. Let $E_{S}^{*}$, $E_{S S}^{*}$, and $E_{M}^{*}$ denote a star, Steiner star, and matching of minimal, minimal, and maximal length, respectively. For easier notation, we write $L_{S}, L_{S S}$, and $L_{M}$ for $\left\|E_{S}^{*}\right\|,\left\|E_{S S}^{*}\right\|$, and $\left\|E_{M}^{*}\right\|$; furthermore, we denote by $\rho(X, Y)$ (with $X, Y=S, S S, M$ ) the worst-case ratio $\sup _{P \in R^{d}} L_{X} / L_{Y}$.

Before we consider various geometric instances, we note a general bound on ratios that holds for all weight functions on the edges, even if we do not have triangle inequality. 
Observation 1. For arbitray weighted graphs $G$, we have $\rho(S, M)=2$.

Proof. Let matrix $A$ be the distance matrix of the points in $P$, so $A(i, j)$ is the distance between $p_{i}$ and $p_{j}$. Let $S$ be the sum of all entries in $A$. Since $L_{S}$ is the minimal row sum of $A$, we have by the pigeonhole principle that $L_{S} \leq S / n$. The maximal matching consists of $n / 2$ elements of $A$, so again by the pigeonhole principle we have $L_{M} \geq S /(2 n)$. Hence,

$$
L_{S} \leq S / n \leq 2 L_{M} .
$$

To see that the ratio $L_{S} / L_{M}$ can be arbitrarily close to 2 , even if we assume triangle inequality, consider the complete graph on $n$ vertices, with all edge weights being 1 . Then $L_{S}=(n-1)$, and $L_{M}=n / 2$.

For the value $\rho(S, S S)$, we note the following:

Observation 2. Assuming triangle inequality, we have $\rho(S, S S)=2$. Without triangle inequality, $\rho(S, S S)$ is unbounded.

Proof. Let $c$ be the center of an optimal Steiner star. Let $v_{0} \in V$ be a vertex closest to $c$, and let $w\left(c, v_{0}\right)=d$. Then by triangle inequality, $w\left(v_{0}, v_{i}\right) \leq w\left(v_{0}, c\right)+w\left(c, v_{i}\right)$, so the $\operatorname{star} S t\left(v_{0}\right)$ with center $v_{0}$ satisfies $L_{S} \leq\left\|S t\left(v_{0}\right)\right\| \leq(n-1) d+L_{S S} \leq 2 L_{S S}$.

To see that the bound of 2 is tight, let $G$ be the complete graph on $n$ vertices, with all edge weights being 2 . Let $\bar{G}=(V \cup\{c\}, \bar{E})$ be a complete graph on $(n+1)$ vertices, with all edges adjacent to $c$ having weight 1 . Then $L_{S}=2(n-1)$ and $L_{S S}=n$.

For a class of examples without triangle inequality and unbounded $\rho(S, S S)$, let $G=(V, E)$ be the complete graph on $n$ vertices, with each edge having weight $2 N$. Let $\bar{G}=(V \cup\{c\}, \bar{E})$ be the complete graph on $(n+1)$ vertices, with all edges adjacent to $c$ having weight 1 . Then $L_{S}=2 N(n-1)>N n=N L_{S S}$.

In a geometric setting, distances in an arrangement of points are far more restricted, so the above bounds may no longer be best possible. It is the main purpose of this paper to provide tight estimates for geometric scenarios.

\section{Euclidean Distances in Two-Dimensional Space}

Throughout this and the following section we consider arrangements of points in twoand three-dimensional space, with distances measured according to the Euclidean metric. At several occasions, we make use of the following lemma:

Lemma 1. Given a triangle with edge lengths $a, b$, and $c$, where the angle $\gamma$ opposite $c$ is bounded from below by $\varphi>0$. Then we have $a+b \leq \sqrt{2 / 1-\cos \varphi} \cdot c$. For the special case $\varphi=2 \pi / 3$, we get $a+b \leq 2 / \sqrt{3} \cdot c$; for $\varphi=\pi / 2$, we get $a+b \leq \sqrt{2} \cdot c$.

Proof. By triangle inequality, we have $a+b \geq c$, so $(a+b)^{2} / c^{2}$ is bounded from below by 1 . By the cosine theorem, we have $(a+b)^{2} / c^{2}=(a+b)^{2} /\left(a^{2}+b^{2}-2 a b \cos \gamma\right)$. 
This expression is symmetric in $a$ and $b$ and attains the lower bound of 1 for $a=0$ or $b=0$, and the maximum is attained for $a=b$, from which the claim follows.

\subsection{Minimum Steiner Stars and Maximum Matchings}

In this subsection we give a proof of Suri's conjecture [16]. Throughout the section, distances are measured according to the Euclidean metric. The key idea is to make use of Lemma 1 and construct a matching that guarantees that the angle between the connections of the matched points and the origin has a good lower bound.

Let $l$ be a directed line in the plane. We say that $l$ is a halving line of $P$ if at most half of the points of $P$ are to the right of $l$ and at most half of the points of $P$ are to the left of $l$.

Lemma 2. For point sets $P$ in two-dimensional space we can find three directed lines $l_{0}, l_{1}$, and $l_{2}$ such that the three lines intersect in a common point, all three lines are halving lines of $P$ and the smallest angle between any two lines is $\pi / 3$.

Proof. See Fig. 1. The collection of halving lines for a given direction $\alpha$ form a directed closed strip which we call $S_{\alpha}$. Consider the strips $S_{0}, S_{\pi / 3}$, and $S_{2 \pi / 3}$. If these three strips have a point in common, we are done. Therefore assume without loss of generality that $S_{0} \cap S_{\pi / 3}$ lies to the left of $S_{2 \pi / 3}$. It follows that $S_{\pi} \cap S_{4 \pi / 3}$ lies to the right of $S_{5 \pi / 3}$.

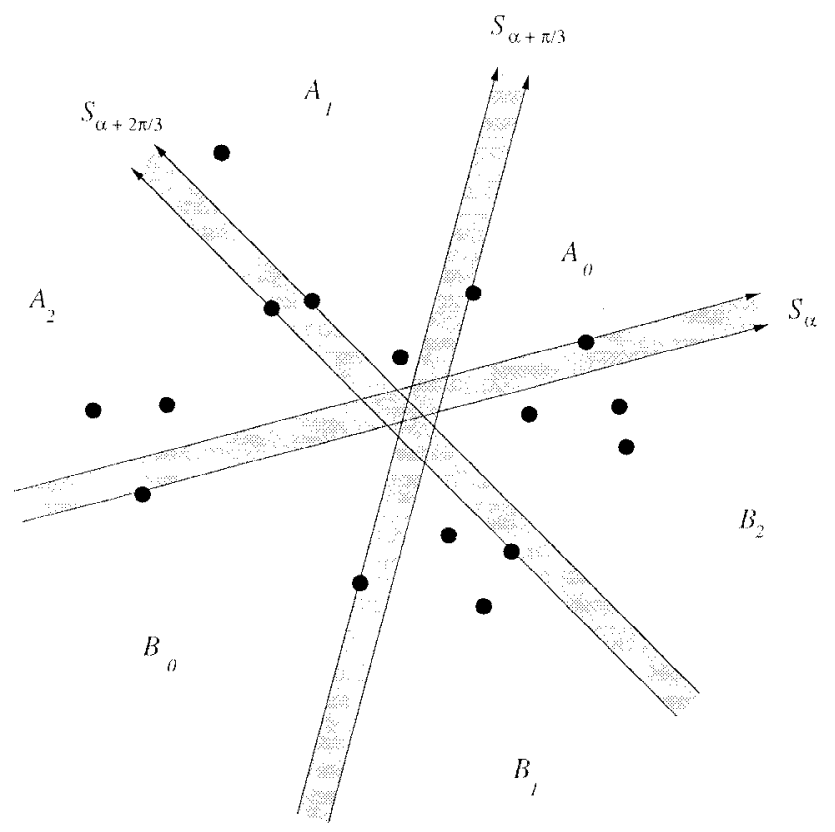

Fig. 1. Finding a small Steiner star and a large matching. 
We now consider the strips $S_{\alpha}, S_{\alpha+\pi / 3}$, and $S_{\alpha+2 \pi / 3}$, where $\alpha$ increases from $\alpha=0$ to $\alpha=\pi$. The three strips move in a continuous manner. Suppose that for no value of $\alpha$ do the three strips have a point in common. Then $S_{\alpha} \cap S_{\alpha+\pi / 3}$ stays to the left of $S_{\alpha+2 \pi / 3}$, which contradicts the fact that $S_{\pi} \cap S_{4 \pi / 3}$ lies to the right of $S_{5 \pi / 3}$. Therefore there is a value of $\alpha$ for which the three strips $S_{\alpha}, S_{\alpha+\pi / 3}$, and $S_{\alpha+2 \pi / 3}$ have a point in common, which proves the lemma.

Theorem 1. For point sets $P$ of even cardinality in two-dimensional space with Euclidean distances, we have $\rho(S S, M)=2 / \sqrt{3}$.

Proof. The following example with $L_{S S} / L_{M}=2 / \sqrt{3}$ was given by Suri. Suppose $n$ is divisible by 6 . Place $n / 3$ points on each corner of an equilateral triangle with sides of length $2 \sqrt{3}$. Then $L_{S S}=2 n$ and $L_{M}=n \sqrt{3}$.

To see that $\rho(S S, M) \leq 2 / \sqrt{3}$, find three lines $l_{0}, l_{1}$, and $l_{2}$ as shown in Fig. 1 , such that the three lines intersect in a common point, all three lines are halving lines for $P$ and the smallest angle between any two lines is $\pi / 3$. These lines divide $P$ into six sets $A_{0}, A_{1}$, $A_{2}, B_{0}, B_{1}$, and $B_{2}$, where $A_{i}$ lies opposite $B_{i}$ for all $i$, as shown in Fig. 1 . By assigning the points of $P$ on the three lines to only one of the sets it belongs to, we can assume that $\left|A_{0}\right|+\left|A_{1}\right|+\left|A_{2}\right|=n / 2=\left|B_{0}\right|+\left|B_{1}\right|+\left|B_{2}\right|$, as well as $\left|A_{0}\right|+\left|A_{1}\right|+\left|B_{2}\right|=$ $n / 2=\left|B_{0}\right|+\left|B_{1}\right|+\left|A_{2}\right|$, and $\left|A_{0}\right|+\left|B_{1}\right|+\left|B_{2}\right|=n / 2=\left|B_{0}\right|+\left|A_{1}\right|+\left|A_{2}\right|$. This implies $\left|A_{i}\right|=\left|B_{i}\right|$ for all $i$.

Let $O$ be the intersection of $l_{0}, l_{1}$, and $l_{2}$. Construct a Steiner star $E_{S S}$ with $O$ as its center. Now choose an (arbitrary) perfect matching $E_{M}$ of the points in $A_{i}$ with the points in $B_{i}$ for all $i$.

We know that by construction, for each edge $(p, q)$ in $E_{M}$, the angle $\angle(p, O, q)$ is bounded from below by $\varphi=2 \pi / 3$. By Lemma 1 , this implies $\|p-O\|+\|O-q\| \leq$ $2 / \sqrt{3} \cdot\|p-q\|$. So $L_{S S} \leq\left\|E_{S S}\right\| \leq 2 / \sqrt{3} \cdot\left\|E_{M}\right\| \leq 2 / \sqrt{3} \cdot L_{M}$.

\subsection{Minimum Stars and Minimum Steiner Stars}

We turn to the possible values of $\rho(S, S S)$. As we noted in the Introduction, this ratio appears in location problems like the Weber problem, where the sum of distances from a depot to a set of positions is to be minimized. Here we give bounds on the worst-case value of the possible payoff from opening a new depot.

Since $L_{S} \geq L_{S S}$, we assume without loss of generality that the star center $c$ of an optimal Steiner star $E_{S S}^{*}$ is not an element of $P$.

Let the line segments, or rays, of a Steiner star be $r_{i}$, and denote their lengths by $a_{i}$. Let $\alpha_{i}$ be the angle between the positive $x$-axis and ray $r_{i}$.

Lemma 3. For the angles $\alpha_{i}$ of $E_{S S}^{*}$, and any $\theta$, we have the local optimality condition

$$
\sum_{i=0}^{n-1} \cos \left(\alpha_{i}+\theta\right)=0
$$


Proof. For the given Steiner star center, the sum of distances is locally minimal, so the projection of the gradient onto any line must disappear; by linearity of the derivative, $\sum_{i=0}^{n-1} \cos \left(\alpha_{i}+\theta\right)$ is the projection of the derivative onto the line that encloses an angle of $\theta$ with the origin.

Lemma 4. Let $E_{S S}$ be a Steiner star of $P=\left\{p_{0}, \ldots, p_{n-1}\right\}$ and let $a_{i}, r_{i}$, and $\alpha_{i}$ be defined as above. Let $b_{i}$ be the distance between $p_{0}$ and $p_{i}$. In the special case that all points of $P$ lie on a circle centered at $c$, of radius $a_{0}=a_{i}$ for all $i$, the local optimality condition

$$
\sum_{i=0}^{n-1} \cos \left(\alpha_{i}+\theta\right)=0 \quad \text { implies that } \quad \sum_{i=0}^{n-1} b_{i} \leq a_{0} n \sqrt{2}
$$

Proof. We have $\sum_{i=0}^{n-1} \cos \left(\alpha_{i}+\theta\right)=0$ for all $\theta$, so without loss of generality we can assume that $P$ is rotated around the origin so that $p_{0}=\left(a_{0}, 0\right)$. We have

$$
b_{i}=2 a_{0} \sin \left(\frac{\alpha_{i}}{2}\right)=a_{0} \sqrt{2\left(1-\cos \alpha_{i}\right)}
$$

(see also Fig. 2). The function $f(\alpha)=\sqrt{1-\cos \alpha}$, where $0 \leq \alpha<2 \pi$, is a concave function of $\cos \alpha$. By Jensen's inequality [15] we have

$$
\sum_{i=0}^{n-1} \frac{1}{n} \sqrt{1-\cos \alpha_{i}} \leq \sqrt{1-\left(\sum_{i=0}^{n-1} \frac{1}{n} \cos \alpha_{i}\right)}=1,
$$

from which the result follows.

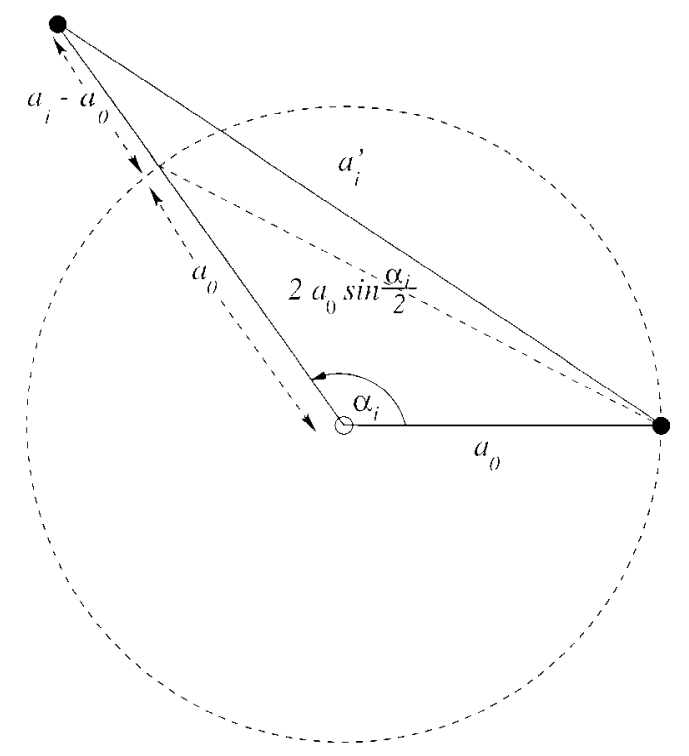

Fig. 2. Length of a chord in a circle. 
Now we can prove the following upper bound:

Theorem 2. For point sets $P$ in two-dimensional space with Euclidean distances, we have $4 / \pi \leq \rho(S, S S) \leq \sqrt{2}$.

Proof. For the lower bound, choose $P$ to be a set of points spaced evenly on the unit circle. Assume that $P$ includes the point $(x, y)=(0,-1)$. The center of $E_{S S}^{*}$ is the center of the circle, so $L_{S S}=n$. Consider the star $E_{S}$ centered at $(0,-1)$. Denote the rays of $E_{S}$ by $r_{i}$ and their lengths by $a_{i}$. Let $\alpha_{i}$ be the angle between the positive $x$-axis and ray $r_{i}$. We have $a_{i}=2 \sin \alpha_{i}$. Therefore the average ray length in the limit is

$$
\frac{1}{\pi} \int_{0}^{\pi / 2} 4 \sin \alpha d \alpha=\frac{4}{\pi} .
$$

Now consider the upper bound. If the center of $E_{S S}^{*}$ is an element of $P$, the bound holds, so assume that the center of the $E_{S S}^{*}$ is not an element of $P$. Without loss of generality assume that the center of $E_{S S}^{*}$ is the origin, that $r_{0}$ is a shortest ray, and that $r_{0}$ runs along the positive $x$-axis, as shown in Fig. 2.

Consider the star $E_{S}$ centered at the endpoint of $r_{0}$. Denote the rays of $E_{S}$ by $r_{i}^{\prime}$ and their length by $a_{i}^{\prime}$. Using triangle inequality we have

$$
a_{i}^{\prime} \leq\left(a_{i}-a_{0}\right)+2 a_{0} \sin \left(\frac{\alpha_{i}}{2}\right) .
$$

So from Lemma 4 we have

$$
\begin{aligned}
L_{S} & \leq\left\|E_{S}\right\|=\sum_{i=0}^{n-1} a_{i}^{\prime} \leq \sum_{i=0}^{n-1} a_{i}-n a_{0}+n a_{0} \sqrt{2} \\
& \leq L_{S S}+L_{S S}(\sqrt{2}-1)=\sqrt{2} \cdot L_{S S} .
\end{aligned}
$$

\subsection{Minimum Stars and Maximum Matchings}

It is not hard to derive upper and lower bounds for $\rho(S, M)$ by using the previous results of this section:

Theorem 3. For point sets $P$ of even cardinality in two-dimensional space with Euclidean distances, we have $\frac{4}{3} \leq \rho(S, M) \leq 2 \sqrt{2} / \sqrt{3}$.

Proof. The upper bound follows immediately from Theorems 1 and 2 .

For the lower bound, suppose $n$ is divisible by 6 , and place $n / 3$ points on each corner of an equilateral triangle with sides of length 6. Then $L_{S}=4 n$ and $L_{M}=3 n$.

\section{Euclidean Distances in Three-Dimensional Space}

Now we turn to point sets in three-dimensional space under Euclidean distances. Again, we start by providing tools for the ratio $\rho(S S, M)$. 
Following an idea similar to the one from Section 3.1, we show that there always exist three orthogonal planes that partition $P$ into eight octants such that opposite octants contain the same number of points.

Let $p$ be a plane. We say that $p$ is a halving plane of $P$ if at most half of the points of $P$ are on one side of $p$ and at most half of the points of $P$ are on the other side of $p$.

Let $p_{0}, p_{1}$, and $p_{2}$ be three orthogonal planes, each of which is a halving plane of $P$. Each plane $\Pi_{i}$ divides $P$ into points above $\Pi_{i}$, on $\Pi_{i}$, and below $\Pi_{i}$. Let $Q_{0}^{i}$ be the points in $P$ below $\Pi_{i}$ and let $Q_{1}^{i}$ be the points in $P$ above $\Pi_{i}$. We assign the points on $\Pi_{i}$ to either $Q_{0}^{i}$ and $Q_{1}^{i}$ in such a way that $\left|Q_{0}^{i}\right|=\left|Q_{1}^{i}\right|$. We call $\left|Q_{0}^{i}\right|$ and $\left|Q_{1}^{i}\right|$ the set of points below and above $\Pi_{i}$, respectively, even though some of these points may in fact lie on $\Pi_{i}$. We define, for $i, j, k \in\{0,1\}$,

$$
Q_{i j k}=Q_{i}^{2} \cap Q_{j}^{1} \cap Q_{k}^{0}
$$

For example, $Q_{110}$ is the set of points in $P$ above $\Pi_{2}$, above $\Pi_{1}$, and below $\Pi_{0}$. Since $\left|Q_{0}^{i}\right|=\left|Q_{1}^{i}\right|$, we can derive the following equalities:

$$
\begin{aligned}
\left|Q_{000}\right|+\left|Q_{001}\right| & =\left|Q_{110}\right|+\left|Q_{111}\right|, \\
\left|Q_{100}\right|+\left|Q_{101}\right| & =\left|Q_{010}\right|+\left|Q_{011}\right|, \\
\left|Q_{000}\right|+\left|Q_{010}\right| & =\left|Q_{101}\right|+\left|Q_{111}\right|, \\
\left|Q_{001}\right|+\left|Q_{011}\right| & =\left|Q_{100}\right|+\left|Q_{110}\right|, \\
\left|Q_{000}\right|+\left|Q_{100}\right| & =\left|Q_{011}\right|+\left|Q_{111}\right|, \\
\left|Q_{001}\right|+\left|Q_{101}\right| & =\left|Q_{010}\right|+\left|Q_{110}\right| .
\end{aligned}
$$

We show that we can always find three orthogonal planes such that opposite octants, i.e., $Q_{i j k}$ and $Q_{(1-i)(1-j)(1-k)}$ have the same cardinality.

Lemma 5. For point sets $P$ in three-dimensional space, we can find three orthogonal planes such that $\left|Q_{i j k}\right|=\left|Q_{(1-i)(1-j)(1-k)}\right|$ for all $i, j, k$.

Proof. Notice that it suffices to find three orthogonal planes such that $\left|Q_{000}\right|=\left|Q_{111}\right|$, since this implies that $\left|Q_{i j k}\right|=\left|Q_{(1-i)(1-j)(1-k)}\right|$ for all $i, j, k$. We first assume that the points are in general position, in the sense that if we project all points in $P$ onto the $(z=0)$ plane, then no three points are collinear and no line through two points is perpendicular to another line through two points. Let $\Pi_{2}$ be a halving plane parallel to the $(z=0)$ plane. We map all points from $P$ onto the $(z=0)$ plane, and call the projected points from $Q_{1}^{2}$ and $Q_{0}^{2}$ the black and white points, respectively.

The problem is now a two-dimensional one. Orthogonal halving planes $\Pi_{0}$ and $\Pi_{1}$ will become halving lines $l_{0}$ and $l_{1}$ in the projection. Let a directed line $l$ be a halving line if at most half of the black and white points are to the left of $l$ and at most half lie to the right of $l$. Because of the nondegeneracy assumption there are at most two points on a halving line $l$. The collection of halving lines for a given direction $\alpha$ form a directed closed strip which we call $S_{\alpha}$. Let $l_{\alpha}$ be the halving line in the middle of $S_{\alpha}$. Consider the strips $S_{\alpha}$ and $S_{\alpha+\pi / 2}$. The corresponding lines $l_{\alpha}$ and $l_{\alpha+\pi / 2}$ divide the points into four subsets $Q_{i j}$ for $i, j \in\{0,1\}$, where $Q_{i j}$ is the projection of $Q_{0 i j} \cup Q_{1 i j}$. Therefore $\left|Q_{00}\right|=\left|Q_{11}\right|$ and $\left|Q_{01}\right|=\left|Q_{10}\right|$, as illustrated in Fig. 3 . 


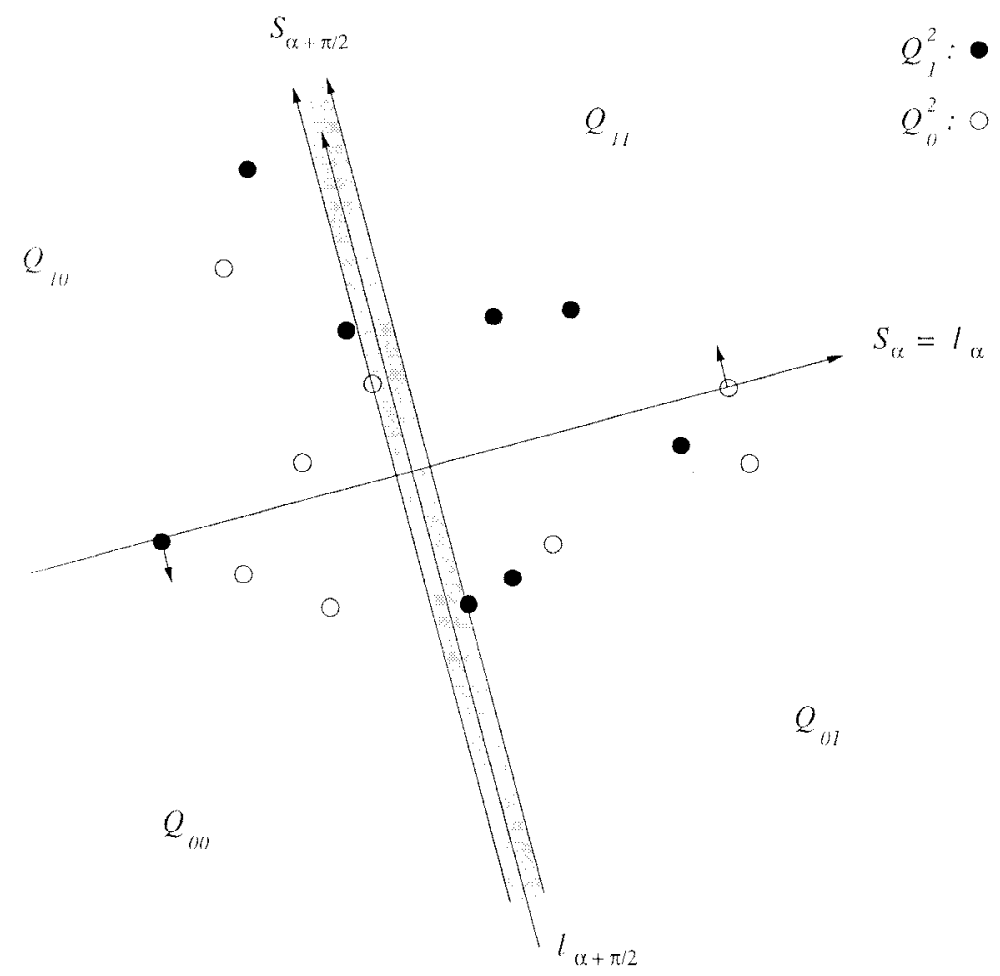

Fig. 3. Orthogonal halving strips.

As $\alpha$ increases, at most one point is added to and at most one point is removed from $Q_{i j}$ at any one time. Changes only occur when $S_{\alpha}$ or $S_{\alpha+\pi / 2}$ is a line. Because of the nondegeneracy assumption it is not possible that both $S_{\alpha}$ and $S_{\alpha+\pi / 2}$ are lines. Either

- the number of points in two opposite quadrants such as $Q_{00}$ and $Q_{11}$ both increase or both decrease, or

- two neighboring quadrants exchange a point.

In the first case, $\left|Q_{000}\right|$ and $\left|Q_{111}\right|$ either both increase, both decrease, or only one changes. It is not possible for $\left|Q_{000}\right|$ to decrease and for $\left|Q_{111}\right|$ to increase or vice versa. In the latter case, at most one of $\left|Q_{000}\right|$ and $\left|Q_{111}\right|$ changes. Therefore, if for some value of $\alpha_{0}$ we have $\left|Q_{000}\right|<\left|Q_{111}\right|$, while for $\alpha_{1}$ we have $\left|Q_{000}\right|>\left|Q_{111}\right|$, then there is an $\alpha$ with $\alpha_{0}<\alpha<\alpha_{1}$ for which $\left|Q_{000}\right|=\left|Q_{111}\right|$.

Consider first the strips $S_{0}$ and $S_{\pi / 2}$. If $\left|Q_{000}\right|=\left|Q_{111}\right|$, then we are done. Therefore suppose that $\left|Q_{000}\right|<\left|Q_{111}\right|$. It follows that for strips $S_{\pi}$ and $S_{3 \pi / 2}$ we have $\left|Q_{000}\right|>$ $\left|Q_{111}\right|$. Therefore there is a value of $\alpha$ with $0<\alpha<\pi$ for which $\left|Q_{000}\right|=\left|Q_{111}\right|$.

If the nondegeneracy assumption does not hold, we can move all points by an infinitesimal small distance, in such a way that the assumption does hold. The construction shown above gives three orthogonal halving planes of the perturbed set. The same planes 
partition $P$ in the correct way, whereby the perturbation of a point that lies on a halving plane determines to which sides of the plane this point should be assigned.

Theorem 4. For sets of points $P$ in three-dimensional space with Euclidean distances, we have the inequality $\sqrt{3} / \sqrt{2} \leq \rho(S S, M) \leq \sqrt{2}$.

Proof. For the lower bound, suppose $n$ is divisible by 4. Place $n / 4$ points on each corner of a tetrahedron with sides of length 2 . Then $L_{S S}=n \sqrt{3} / \sqrt{2}$ and $L_{M}=n$.

To prove the upper bound, find three orthogonal halving planes that satisfy the condition $\left\|Q_{i j k}\right\|=\left\|Q_{(1-i)(1-j)(1-k)}\right\|$ for all $i, j, k$. Let $O$ be the point of intersection of these three planes. Construct a Steiner star $E_{S} S$ with $O$ as its center. Construct a matching $E_{M}$ by connecting points in each octant to points in the opposite octant.

For each edge $(p, q)$ in $E_{M}$, the angle between the ray from $O$ to $p$ and the ray from $O$ to $q$ is bounded from below by $\pi / 2$, from which we derive by Lemma 1 that $\|p-O\|+\|O-q\| \leq \sqrt{2} \cdot\|p-q\|$. So $L_{S S} \leq\left\|E_{S S}\right\| \leq \sqrt{2} \cdot\left\|E_{M}\right\| \leq \sqrt{2} \cdot L_{M}$.

Next we discuss the worst-case value $\rho(S, S S)$.

Theorem 5. For sets of points $P$ in three-dimensional space with Euclidean distances, we have the inequality $\frac{4}{3} \leq \rho(S, S S) \leq \sqrt{2}$.

Proof. The proof for the upper bound is the same as the proof for the two-dimensional case shown in Theorem 2.

To see the lower bound, let $P$ be a set of points evenly distributed over the unit sphere. Assume $P$ includes the point $(x, y, z)=(0,-1,0)$. The center of $E_{S S}^{*}$ is the center of the sphere, so $L_{S S}=n$. Consider the star $E_{S}$ centered at the point $(0,-1,0)$. Denote the rays of $E_{S}$ by $r_{i}$ and their lengths by $a_{i}$. Let $\alpha_{i}$ be the angle between the $(y=0)$ plane and ray $r_{i}$. We have $a_{i}=2 \sin \alpha_{i}$.

The average ray length can be computed as follows. Let $\theta$ be the angle between a ray and the $(y=0)$ plane and let $\varphi$ be the angle between the projection of the ray onto the $(y=0)$ plane and the positive $x$-axis. If $\Delta \theta=\theta_{1}-\theta_{0}$ and $\Delta \varphi=\varphi_{1}-\varphi_{0}$ are small, then the surface area covered by all rays with angles in this range is approximately equal to $4 \sin \theta \cos \theta \Delta \varphi \Delta \theta$, so the area of the surface of the sphere is

$$
\int_{0}^{\pi / 2} \int_{0}^{2 \pi} 4 \sin \theta \cos \theta d \varphi d \theta=4 \pi
$$

and the average ray length is

$$
\frac{1}{4 \pi} \int_{0}^{\pi / 2} \int_{0}^{2 \pi}(4 \sin \theta \cos \theta)(2 \sin \theta) d \varphi d \theta=\frac{4}{3} .
$$

We conclude our discussion on Euclidean distances:

Observation 3. For point sets in three-dimensional space with Euclidean distances, $\frac{3}{2} \leq \rho(S, M) \leq 2$. 
Proof. We know from Observation 1 that $\rho(S, M)$ cannot exceed 2. For the lower bound, suppose $n$ is divisible by 8 . Place $n / 4$ points on each corner of a tetrahedron with sides of length 4 . Then $L_{S}=3 n$ and $L_{M}=2 n$.

\section{Manhattan Distances in Two-Dimensional Space}

\subsection{Minimum Steiner Stars and Maximum Matchings}

Independent from our work, the following proposition was also noted by Tamir and Mitchell [17]. Since some of the steps are of importance for our further results, we give a sketch of the proof.

Proposition 1. For point sets $P$ of even cardinality in two-dimensional space with Manhattan distances, we have $L_{M}=L_{S S}$.

Proof (Sketch). It is not hard to see that the center for an optimal Steiner star must both be a median of the $x$-coordinates and the $y$-coordinates of the points in $P$. Assume without loss of generality that an optimal center is located at $(0,0)$ and consider the numbers $n_{1}, n_{2}, n_{3}$, and $n_{4}$ of points in each of the four quadrants, with points on the boundary of two quadrants assigned in a suitable way. Using $n_{1}+n_{2}=n_{3}+n_{4}$ and $n_{2}+n_{3}=n_{4}+n_{1}$, we get $n_{1}=n_{3}$ and $n_{2}=n_{4}$, i.e., diagonally opposite quadrants must contain the same number of points. This allows us to match points from opposite quadrants. It is straightforward to see that to each edge of the matching, we have a corresponding pair of edges of the Steiner star with the same total length, implying that the total length of the matching is equal to the total length of the Steiner star.

\subsection{Minimum Stars and Minimum Steiner Stars}

In the following, we consider $\rho(S, S S)$. For any point $p_{i}=\left(x_{i}, y_{i}\right) \in P, L_{S}\left(p_{i}\right)$ is the total length of the star centered at $p_{i}$. We denote by $\rho_{n}$ the supremum of the values $L_{S} / L_{S S}$ for point sets of cardinality $n$. Without loss of generality, we may assume that $L_{S S}>0$, and thus $L_{S S}=1$. Furthermore, we may assume that the origin is an optimal Steiner center.

We make use of the following lemma:

Lemma 6. For any $n$, there are point sets for which $L_{S} / L_{S S}$ attains the value $\rho_{n}$.

Proof. For any fixed $n$, the set of point arrangements with $L_{S S}=1$ and optimal Steiner center $O$ is a compact subset of $R^{2 n}$. Since $L_{S}$ is a continuous function on $R^{2 n}$, the claim holds.

Lemma 7. Let $P$ be a set of $n$ points with $L_{S} / L_{S S}=\rho_{n}$. Then, for all $p_{i} \in P$, $L_{S}\left(p_{i}\right)=\rho_{n}$. 
Proof. Suppose there is a point $p_{i}$ with $L_{S}\left(p_{i}\right)>L_{S}$. For sufficiently small $\varepsilon$, replacing $p_{i}$ by the point $p_{i}^{\prime}=(1-\varepsilon) p_{i}$ does not turn $p_{i}^{\prime}$ into an optimal star center. Thus, the replacement reduces $L_{S S}$ by some small $\varepsilon^{\prime}$, but $L_{S}$ by not more than $\varepsilon^{\prime}$. Therefore, the new arrangement has a ratio of at least $\left(L_{S}-\varepsilon^{\prime}\right) /\left(L_{S S}-\varepsilon^{\prime}\right)>L_{S} / L_{S S}=\rho_{n}$, a contradiction.

It is straightforward to see that this implies the following:

Corollary 1. For an arrangement with $L_{S} / L_{S S}=\rho_{n}$, we cannot move a vertex such that $L_{S S}$ decreases by $\varepsilon$ and $L_{S}$ by not more than $\varepsilon$.

Corollary 2. For an arrangement with $L_{S} / L_{S S}=\rho_{n}$, we cannot move a vertex such that $L_{S S}$ remains the same, one or more of the $L_{S}\left(p_{i}\right)$ increase, and none of them decreases.

The following implication allows a further reduction of arrangements that can achieve the worst-case ratio; it will be used for the proof of Lemma 10.

Corollary 3. For any arrangement in two-dimensional space with Manhattan distances and with $L_{S} / L_{S S}=\rho_{n}$, there cannot be two points $p_{1}=\left(x_{1}, y_{1}\right) \neq p_{2}=\left(x_{2}, y_{2}\right)$, such that $p_{1}$ lies between $p_{2}$ and the origin, i.e., $0 \leq\left(x_{1}, y_{1}\right) \leq\left(x_{2}, y_{2}\right)$, or $0 \leq\left(-x_{1}, y_{1}\right) \leq$ $\left(-x_{2}, y_{2}\right)$, or $0 \leq\left(x_{1},-y_{1}\right) \leq\left(x_{2},-y_{2}\right)$, or $0 \leq\left(-x_{1},-y_{1}\right) \leq\left(-x_{2},-y_{2}\right)$.

Proof. In any of the four cases, it is straightforward to see that $L_{S}\left(p_{1}\right)<L_{S}\left(p_{2}\right)$.

The next lemma shows that we may restrict our attention to arrangements with extreme points on the coordinate axes:

Lemma 8. For any arrangement in two-dimensional space with Manhattan distances and with $L_{S} / L_{S S}=\rho_{n}$, any point $p_{i}=\left(x_{i}, y_{i}\right)$ with minimal or maximal $x_{i}$ among the points in $P$ must have $y_{i}=0$. Conversely, minimal or maximal $y_{i}$ implies $x_{i}=0$.

Proof. Without loss of generality consider a point $p_{i}$ with maximal $y_{i}$ and assume $x_{i}>0$. Let $x_{k}=\max \left\{x_{j} \mid j \neq i\right\}$. If $x_{i}-x_{k}=\delta>0$, replace $p_{i}$ by $\left(x_{k}, y_{i}+\delta\right)$; this does not change $L_{S}$ or $L_{S S}$, and allows us to consider without loss of generality the case $x_{i}-x_{k} \leq 0$.

For sufficiently small $\varepsilon$, replace $p_{i}$ by $p_{i}^{\prime}=p_{i}+(-\varepsilon, \varepsilon)$. This does not change $L_{S S}$, increases $L_{S}\left(p_{k}\right)$, and does not decrease $L_{S}\left(p_{j}\right)$ for any $j \neq k$, contradicting Corollary 2.

Lemma 9. For any $n$, we have $\rho_{n} \leq \rho_{k n}$.

Proof. Suppose we have an arrangement of $n$ points with $L_{S} / L_{S S}=\rho_{n}$. Replace each point by $k$ copies; this yields an arrangement of $k n$ points with $L_{S} / L_{S S}=\rho_{n}$. 
Lemma 10. For any arrangement in two-dimensional space with Manhattan distances and with $L_{S} / L_{S S}=\rho_{n}$, there can be at most four points $p_{i}$ with $x_{i}, y_{i} \neq 0$.

Proof. Assume that there are at least five points not on coordinate axes. Then we may assume without loss of generality that two of them (say, $p_{1}=\left(x_{1}, y_{1}\right), p_{2}=\left(x_{2}, y_{2}\right)$ ) are in the positive quadrant. Because of Corollary 3 , we may assume that $0<x_{2}<x_{1}$ and $0<y_{1}<y_{2}$. Define

$$
\begin{aligned}
& n_{0}=\text { the number of vertices } i \text { with } x_{1}<x_{i}, \\
& n_{1}=\text { the number of vertices } i \neq 1,2 \text { with } x_{1} \leq x_{i} \leq x_{2}, \\
& n_{2}=\text { the number of vertices } i \text { with } x_{i}<x_{2}, \\
& m_{0}=\text { the number of vertices } i \text { with } y_{2}<y_{i}, \\
& m_{1}=\text { the number of vertices } i \neq 1,2 \text { with } y_{1} \leq y_{i} \leq y_{2}, \\
& m_{2}=\text { the number of vertices } i \text { with } y_{i}<y_{1} .
\end{aligned}
$$

So $n_{2}>n_{0}$ and $m_{2}>m_{0}$. Let $\varepsilon_{x}$ and $\varepsilon_{y}$ be such that $\varepsilon_{x} / \varepsilon_{y}=\left(m_{2}-m_{0}\right) /\left(n_{2}-n_{0}\right)$. Also let $\varepsilon_{x}$ and $\varepsilon_{y}$ be positive but smaller than the smallest nonzero difference between the $x$-coordinates and $y$-coordinates of any two points, respectively. Now replace $p_{1}$ by $p_{1}^{\prime}=p_{1}+\left(\varepsilon_{x},-\varepsilon_{y}\right)$ and $p_{2}$ by $p_{2}^{\prime}=p_{2}+\left(-\varepsilon_{x},+\varepsilon_{y}\right)$. It is not hard to see that these replacements do not decrease $L_{S}\left(p_{i}\right)$ for any $i \neq 1$, 2. Furthermore, $L_{S S}$ does not change. The value $L_{S}\left(p_{1}\right)$ changes by $\Delta_{1}=\left(n_{2}+n_{1}-n_{0}\right) \varepsilon_{x}+\left(-m_{2}+m_{1}+m_{0}\right) \varepsilon_{y}+2 \varepsilon_{x}+2 \varepsilon_{y}$. So $\Delta_{1}>\left(n_{2}-n_{0}\right) \varepsilon_{x}+\left(-m_{2}+m_{0}\right) \varepsilon_{y}=\left(m_{2}-m_{0}\right) \varepsilon_{y}+\left(-m_{2}+m_{0}\right) \varepsilon_{y}=0$. Similarly, the value $L_{S}\left(p_{2}\right)$ changes by $\left(-n_{2}+n_{1}+n_{0}\right) \varepsilon_{x}+\left(m_{2}+m_{1}-m_{0}\right) \varepsilon_{y}+2 \varepsilon_{x}+2 \varepsilon_{y}$, which is also positive. This contradicts Corollary 2.

In order to analyze the limit of the sequence $\rho_{n}$, we define a sequence $\beta_{n}$. This is the supremum of the values $L_{S} / L_{S S}$ for all arrangements of $n$ points, such that any point lies on a coordinate axis.

With the help of Corollary 3 and Lemmas 9 and10, it is not hard to prove that, for arrangements with many points, the bounded number of points not on coordinate axes becomes negligible for the worst-case ratio:

Lemma 11. $\lim \sup _{n \rightarrow \infty} \rho_{n}=\lim \sup _{n \rightarrow \infty} \beta_{n}$.

Proof. For any $n$, consider a point arrangement $P_{n}$ with $L_{S S}=1$ and $L_{S}=\rho_{n}$. By Lemma 10 , for any $P_{n}$, there can be at most four points not on coordinate axes; by Corollary 3 , we conclude that the points on the axes are positioned at $p_{1}=\left(d_{1}, 0\right)$, $p_{2}=\left(0, d_{2}\right), p_{3}=\left(-d_{3}, 0\right), p_{4}=\left(0,-d_{4}\right)$, with multiplicities $n_{1}, n_{2}, n_{3}, n_{4}$, and $\sum_{i=1}^{4} n_{i} \geq n-4$.

Because of Lemma 9, we are done if there are only finitely many $P_{n}$ with a point not on an axis. So assume there are infinitely many $P_{n}$ with a point $p_{0}=\left(x_{0}, y_{0}\right)>(0,0)$. By Corollary 3, we conclude that, for any such $P_{n}, d_{1}>x_{0}$ and $d_{2}>y_{0}$. If $d_{1}$ and $d_{2}$ tend to zero as $n$ becomes large, the contribution of $p_{0}$ to $L_{S S}$ and $L_{S}$ becomes arbitrarily small, and we are done. 
So suppose without loss of generality that $d_{1}=\max \left\{d_{j} \mid j=1, \ldots, 4\right\}$ and that $d_{1}$ remains bounded from below. Since $L_{S S}=1$, this means that $n_{1}$ remains bounded. As $n$ becomes large, it follows that some $n_{i}$ become arbitrarily large. Then $L_{S S}=1$ implies that $d_{i}$ tends to zero. Consider the star $E_{S(j)}$ centered at $p_{j}$. Using 0 as a lower and $3 d_{1}$ as an upper bound for the distance of points not on axes to $p_{1}$ and $p_{i}$, respectively, we get

$$
\left\|E_{S(1)}\right\| \geq n_{2} d_{2}+n_{3} d_{3}+n_{4} d_{4}+\left(n_{2}+n_{3}+n_{4}\right) d_{1}=\sum_{j=1}^{4} n_{j} d_{j}+\left(\sum_{j=1}^{4} n_{j}-2 n_{1}\right) d_{1},
$$

whereas

$$
\left\|E_{S(i)}\right\| \leq 12 d_{1}+\sum_{j=1}^{4} n_{j} d_{j}+\left(\sum_{j=1}^{4} n_{j}-2 n_{i}\right) d_{i} .
$$

This means that, for sufficiently large $n$, we have

$$
\left\|E_{S(1)}\right\|-\left\|E_{S(i)}\right\| \geq\left(d_{1}-d_{i}\right) \sum_{j=1}^{4} n_{j}-2 n_{1} d_{1}+2 n_{i} d_{i}-12 d_{1}>0,
$$

since $\left(d_{1}-d_{i}\right) \sum_{j=1}^{4} n_{j}$ gets arbitrarily large for increasing $n$, while all other terms are bounded from below. This contradicts Lemma 7, and we are done.

In order to establish an upper bound of the sequence $\beta_{n}$, we need the following lemma:

Lemma 12. Let $0 \leq \lambda_{1}, \ldots, \lambda_{2 d}<\frac{1}{2}$, such that $\sum_{i=1}^{2 d} \lambda_{i}=1$. Then

$$
\sum_{i=1}^{2 d} \frac{\lambda_{i}}{1-2 \lambda_{i}} \geq \frac{d}{d-1}
$$

Proof. Since $f(x)=x /(1-2 x)$ is a convex function on the interval $\left[0, \frac{1}{2}\right)$, we have from Jensen's inequality [15]

$$
f\left(\sum_{i=1}^{2 d} \frac{1}{2 d} \lambda_{i}\right) \leq \frac{1}{2 d} \sum_{i=1}^{2 d} f\left(\lambda_{i}\right)
$$

So

$$
\frac{1}{2 d-2} \leq \frac{1}{2 d} \sum_{i=1}^{2 d} \frac{\lambda_{i}}{1-2 \lambda_{i}}
$$

from which the lemma follows.

Now we can proceed to prove the following:

Theorem 6. For point sets $P$ in two-dimensional space with Manhattan distances, we have $\rho(S, S S)=\frac{3}{2}$. 
Proof. To see the lower bound, suppose $n$ is divisible by 4 . Place $n / 4$ points on each corner of the points $(1,0),(0,1),(-1,0),(0,-1)$. Then $L_{S}=3 n / 2$ and $L_{S S}=n$.

By Lemma 11, we only have to show that $\beta_{n} \leq \frac{3}{2}$ for the upper bound. Similar to Lemma 6, we can assume that there are sets of $n$ points for which $L_{S} / L_{S S}=\beta_{n}$. By Lemma 3 , these sets consist of $n_{1}$ points $p_{1}$ at position $\left(d_{1}, 0\right)$, of $n_{2}$ points $p_{2}$ at position $\left(0, d_{2}\right)$, of $n_{3}$ points $p_{3}$ at position $\left(-d_{3}, 0\right)$, and of $n_{4}$ points $p_{4}$ at position $\left(0,-d_{4}\right)$, with $d_{i} \geq 0$. We assume that

$$
L_{S S}=\sum_{i=1}^{4} n_{i} d_{i}=1
$$

Furthermore, $L_{S}\left(p_{i}\right)=\sum_{j \neq i} n_{j}\left(d_{i}+d_{j}\right)=\left(\sum_{j=1}^{4} n_{j} d_{j}\right)+\left(\sum_{j=1}^{4} n_{j} d_{i}\right)-2 n_{i} d_{i}=$ $1+\left(n-2 n_{i}\right) d_{i}$. By Lemma 7, we have $L_{S}\left(p_{i}\right)=\beta_{n}$, which implies

$$
d_{i}=\frac{\beta_{n}-1}{\left(n-2 n_{i}\right)} .
$$

Equations (1) and (2) yield

$$
\frac{1}{\beta_{n}-1}=\sum_{i=1}^{4} \frac{n_{i}}{\left(n-2 n_{i}\right)}
$$

With $n_{i} / n=\lambda_{i}$, Lemma 12 implies $1 /\left(\beta_{n}-1\right) \geq 2$, so $\beta_{n} \leq \frac{3}{2}$, and we are done.

Together with Theorem 1, this implies the following:

Corollary 4. For point sets $P$ of even cardinality in two-dimensional space with Manhattan distances, we have $\rho(S, M)=\frac{3}{2}$.

\section{Manhattan Distances in Three-Dimensional Space}

It was noted by Tamir and Mitchell in [17] that the equality $L_{M}=L_{S S}$ that holds in two-dimensional rectilinear space is no longer valid in three-dimensional space. In fact, their example $P=\{(1,1,1),(1,-1,-1),(-1,1,-1),(-1,-1,1)\}$ shows that $\rho(S S, M) \geq \frac{3}{2}$. The following result shows that this is a worst-case example:

Theorem 7. For point sets $P$ of even cardinality in three-dimensional space with Manhattan distances, we have $\rho(S S, M)=\frac{3}{2}$.

Proof. Assume that $O=(0,0,0)$ is the center of an optimal Steiner star, so $\mid\left\{p_{i} \mid x_{i}<\right.$ $0\}|\leq n / 2|,\left\{p_{i} \mid x_{i}>0\right\}|\leq n / 2|,\left\{p_{i} \mid y_{i}<0\right\} \mid \leq n / 2$, etc. Then $L_{S S}=\sum_{i}\left|x_{i}\right|+$ $\left|y_{i}\right|+\left|z_{i}\right|$. Without loss of generality, assume that $\sum_{i}\left|z_{i}\right| \leq \sum_{i}\left|x_{i}\right| \leq \sum_{i}\left|y_{i}\right|$. Now consider the point set $P^{\prime}=\left\{p_{i}^{\prime} \mid i=1, \ldots, n\right\}$, where $p_{i}^{\prime}=\left(x_{i}, y_{i}, 0\right)$. Because of the above conditions, $O$ is the center of an optimal Steiner star $E_{S S}^{*^{\prime}}$ for $P^{\prime}$. We have 
$\left\|E_{S S}^{*^{\prime}}\right\|=\sum_{i}\left|x_{i}\right|+\left|y_{i}\right| \geq 2 / 3 L_{S S}$. By Proposition $1, P^{\prime}$ has a matching $E_{M}^{\prime}$ of size $\left\|E_{S S}^{*^{\prime}}\right\|$, and the claim follows.

Using the same tools as in the two-dimensional case, we can show the following for $\rho(S, S S)$ :

Theorem 8. For point sets $P$ in three-dimensional space with Manhattan distances, we have $\rho(S S, S)=\frac{5}{3}$.

Proof. For a lower bound, suppose $n$ is divisible by 6 . Place $n / 6$ points on each of the points $(1,0,0),(0,1,0),(0,0,1),(-1,0,0),(0,-1,0),(0,0,-1)$. Then $L_{S}=5 n / 3$ and $L_{S S}=n$.

For the upper bound we proceed similarly to the proof of Theorem 6 . Note that Lemmas 6 and 7 and Corollaries 1 and 2 stay valid without any change, as well as Lemmas 9 and 12. It is straightforward to modify Corollary 3 and Lemma 8 to higher dimensions. Lemma 10 is replaced by the following three-dimensional version:

For any arrangement with $L_{S} / L_{S S}=\rho_{n}$, there can be at most eight points $p_{i}$ not on coordinate axes.

This is shown as follows: Suppose there are nine points not on coordinate axes, then there must be at least two points $p_{1}=\left(x_{1}, y_{1}, z_{1}\right)$ and $p_{2}=\left(x_{2}, y_{2}, z_{2}\right)$ in the same octant, say the positive one. By the analogue of Corollary 3 , we cannot have $0 \leq\left(x_{1}, y_{1}, z_{1}\right) \leq\left(x_{2}, y_{2}, z_{2}\right)$ or $0 \leq\left(x_{2}, y_{2}, z_{2}\right) \leq\left(x_{1}, y_{1}, z_{1}\right)$ with $p_{1} \neq p_{2}$. This allows us to consider without loss of generality $0<x_{1}<x_{2}, 0<y_{2}<y_{1}$ and apply the same modification to the $x$-and $y$-coordinates of $p_{1}$ and $p_{2}$ as in the proof of Lemma 10.

With the help of these lemmas, the claim of Lemma 11 still holds. Using Lemma 12 for $d=3$, we get

$$
\frac{1}{\beta_{n}-1}=\sum_{i=1}^{6} \frac{n_{i}}{\left(n-2 n_{i}\right)} \geq \frac{d}{d-1}=\frac{3}{2},
$$

implying $\beta_{n} \leq \frac{5}{3}$. This concludes the proof.

For $\rho(S, M)$, we only have the following easy observation:

Observation 4. For point sets $P$ of even cardinality in three-dimensional space under Manhattan distances, we have $\frac{5}{3} \leq \rho(S, M) \leq 2$.

Proof. The upper bound follows from Observation 1. For the lower bound, suppose $n$ is divisible by 6 . Place $n / 6$ points on each of the points $(1,0,0),(0,1,0),(0,0,1)$, $(-1,0,0),(0,-1,0),(0,0,-1)$. Then $L_{S}=5 n / 3$ and $L_{M}=n$. 
Table 1. Lower and upper bounds for worst-case ratios.

\begin{tabular}{lcccc}
\hline Distances & Dimension & Ratio & Lower bound & Upper bound \\
\hline Euclidean & Two & $\rho(S S, M)$ & $\frac{2}{\sqrt{3}}=1.15 \ldots$ & $\frac{2}{\sqrt{3}}=1.15 \ldots$ \\
& & $\rho(S, S S)$ & $\frac{4}{\pi}=1.27 \ldots$ & $\sqrt{2}=1.41 \ldots$ \\
& $\rho(S, M)$ & $\frac{4}{3}=1.33 \ldots$ & $\frac{2 \sqrt{2}}{\sqrt{3}}=1.63 \ldots$ \\
& \multirow{3}{*}{ Three } & $\rho(S S, M)$ & $\frac{\sqrt{3}}{\sqrt{2}}=1.22 \ldots$ & $\sqrt{2}=1.41 \ldots$ \\
& & $\rho(S, S S)$ & $\frac{4}{3}=1.33 \ldots$ & $\sqrt{2}=1.41 \ldots$ \\
& & $\rho(S, M)$ & $\frac{3}{2}=1.5$ & 2 \\
& \multirow{3}{*}{ Twanhattan } & $\rho(S S, M)$ & 1 & 1 \\
& & $\rho(S, S S)$ & $\frac{3}{2}=1.5$ & $\frac{3}{2}=1.5$ \\
& & $\rho(S, M)$ & $\frac{3}{2}=1.5$ & $\frac{3}{2}=1.5$ \\
& \multirow{2}{*}{ Three } & $\rho(S S, M)$ & $\frac{3}{2}=1.5$ & $\frac{3}{2}=1.5$ \\
& & $\rho(S, S S)$ & $\frac{5}{3}=1.66 \ldots$ & $\frac{5}{3}=1.66 \ldots$ \\
& & $\rho(S, M)$ & $\frac{5}{3}=1.66 \ldots$ & 2 \\
\hline
\end{tabular}

\section{Conclusion}

We have derived a number of upper and lower bounds for the largest possible value of the ratios between the size of minimum stars, minimum Steiner stars, and maximum matchings. A summary of our bounds is given in Table 1. Some of these bounds are not tight; in all cases, we suspect that the upper bounds can be improved to match the lower bounds. This belief is strengthened by the fact that some of the tools we used for the case of Manhattan distances (in particular, Lemma 7) are true for Euclidean distances as well. We note the following conjectures:

Conjecture 1. For point sets $P$ in two-dimensional space with Euclidean distances, we have $\rho(S, S S)=4 / \pi$.

Conjecture 2. For point sets $P$ in three-dimensional space with Euclidean distances, we have $\rho(S, S S)=\frac{4}{3}$.

\section{Acknowledgments}

The problem by Suri was around for a few years before he presented it at the 1998 ACM Symposium on Computational Geometry; we would like to thank Gordon Wilfong (who had heard it from Suri) for first bringing it to the second author's attention in 1996. We thank the referees Joe Mitchell and Subhash Suri for very constructive suggestions that helped to improve this paper. Thanks to Arie Tamir for pointing out his related previous work. 


\section{References}

1. C. Bajaj. The algebraic degree of geometric optimization problems. Discrete \& Computational Geometry, 3 (1988), 177-191.

2. A. I. Barvinok, D. S. Johnson, G. J. Woeginger, and R. Woodroofe. The maximum Traveling Salesman Problem under polyhedral norms. Proceedings of the 6th International Integer Programming and Combinatorial Optimization Conference (IPCO VI). LNCS 1412. Springer-Verlag, Berlin, 1998, pp. 195-201.

3. M. Ben-Or. Lower bounds for algebraic computation trees. Proceedings of the 15th ACM Symposium on the Theory of Computing, 1983, pp. 80-86.

4. M. Blum, R. W. Floyd, V. R. Pratt, R. L. Rivest, and R. E. Tarjan. Linear time bounds for median computations. Proceedings of the 4th ACM Symposium on the Theory of Computing, 1972, pp. 119-124.

5. Z. Drezner (ed.). Facility Location. A Survey of Applications and Methods. Springer-Verlag, New York, 1995.

6. D.-Z. Du and F. K. Hwang. A proof of the Gilbert-Pollak conjecture on the Steiner ratio. Algorithmica, 7 (1992), 121-135.

7. J. Edmonds. Maximum matching and a polyhedron with 0, 1-vertices. Journal of Research of the National Bureau of Standards (B), 69 (1965), 69-87.

8. S. P. Fekete. Simplicity and hardness of the Maximum Traveling Salesman Problem under geometric distances. Proceedings of the ACM/SIAM Symposium on Discrete Algorithms, 1999, pp. 337-345.

9. S. P. Fekete and H. Meijer. On minimum stars, minimum Steiner stars, and maximum matchings. Proceedings of the 15th ACM Symposium on Computational Geometry, 1999, pp. 217-226.

10. J. A. Fingerhut, S. Suri, and J. S. Turner. Designing least-cost nonblocking broadband networks. Journal of Algorithms, 24 (1997), 287-309.

11. M. R. Garey and D. S. Johnson. Computers and Intractability. A Guide to the Theory of NP-Completeness. Freeman, New York, 1979.

12. A. J. Goldmann. Optimal center location in simple networks. Transportation Science, 5 (1971), $212-221$.

13. F. K. Hwang, D. S. Richards, and P. Winter. The Steiner Tree Problem. Elsevier Science, Amsterdam, 1992.

14. O. Kariv and L. S. Hakimi. An algorithmic approach to network location problems. II: The p-medians. SIAM Journal on Applied Mathematics, 37 (1979), 539-560.

15. M. Spivak. Calculus. Benjamin, Menlo Park, CA, 1967.

16. S. Suri. Problem \# 5. Problem session of the 14th ACM Symposium on Computational Geometry, 1998. http://www.cs.duke.edu/pankaj/scg98-openprobs/ open-probs.html.

17. A. Tamir and J. S. B. Mitchell. A maximum $b$-matching problem arising from median location models with applications to the roommates problem. Mathematical Programming, 80(2) (1998), 171-194.

Received January 1, 1999, and in revised form July 15, 1999. 